\title{
Optimal Kalman Filtering for a Class of State Delay Systems with Randomly Multiple Sensor Delays
}

\author{
Dongyan Chen and Long Xu \\ Department of Applied Mathematics, Harbin University of Science and Technology, Harbin 150080, China \\ Correspondence should be addressed to Long Xu; longxu19880319@gmail.com
}

Received 17 March 2014; Accepted 29 March 2014; Published 24 April 2014

Academic Editor: Hamid Reza Karimi

Copyright ( 2014 D. Chen and L. Xu. This is an open access article distributed under the Creative Commons Attribution License, which permits unrestricted use, distribution, and reproduction in any medium, provided the original work is properly cited.

\begin{abstract}
The optimal Kalman filtering problem is investigated for a class of discrete state delay stochastic systems with randomly multiple sensor delays. The phenomenon of measurement delay occurs in a random way and the delay rate for each sensor is described by a Bernoulli distributed random variable with known conditional probability. Based on the innovative analysis approach and recursive projection formula, a new linear optimal filter is designed such that, for the state delay and randomly multiple sensor delays with different delay rates, the filtering error is minimized in the sense of mean square and the filter gain is designed by solving the recursive matrix equation. Finally, a simulation example is given to illustrate the feasibility and effectiveness of the proposed filtering scheme.
\end{abstract}

\section{Introduction}

Over the past decades, the estimation/filtering problems have received considerable attention due to their wide application in many practical systems [1-5]. Accordingly, the optimal state estimation/filtering problems have been one of the mainstream research topics in the signal processing field and a large number of results have been reported to design the optimal estimators; see, for example, [6-9]. Generally speaking, the aim of the optimal state estimation is to estimate the internal state of a dynamic system based on the available measurement data and the estimation principle is in the sense of the minimum mean square error (MMSE). Note that the dynamic behaviours of the engineering systems are described by the internal state variable of the systems. Hence, it is important to design the filter so as to further understand and control a practical system and then achieve the desired performance requirements [10-12]. It is worth mentioning that, based on the MMSE principle and the projection theory, the famous Kalman filter has been constructed in [13] for linear discrete stochastic systems and the recursive estimation method has been developed. Subsequently, many important results have been published based on this pioneering method. Moreover, some effective yet easy-to-implement filtering algorithms have been developed in [14, 15] for complex dynamical systems with the prevalently network-induced phenomena.

It is well known that the time delay is inevitable in many industrial process systems [16-22]. Also, it is necessary to deal with the time delay to improve the control performance for the practical systems [23-26]. In the past years, a great deal of effort has been devoted to address the problems of the optimal state estimation for time delay systems. To mention a few, by applying the state augmentation approach, the problem of the optimal state estimation has been investigated in [17] for linear discrete stochastic systems with measurement delay. In [19-22], the optimal filters have been designed for linear state delay systems. In particular, by using the state augmentation approach, the optimal filter has been designed in [19]. Without using the state augmentation approach, a new optimal filter has been constructed in [20] for linear discrete state delay stochastic systems by applying the projection theory and recursive projection formula. It should be noted that the dimension of the proposed filter in [20] is the same as the original system state and then the computational burden can be reduced. Based on the method in [20], the problem of optimal filtering has been studied in [21] for linear discrete state delay systems under uncertain observations. In [22], an 
effective robust Kalman filter has been designed for a class of uncertain state delay systems with random observation delays and missing measurements.

Due to the sudden changes in the environment and unreliability of the communication network, the sensor measurement of the system may experience the unexpected measurement delays in reality [27-29]. However, it is worthwhile mentioning that most of the published results have tackled the deterministic delays only. In fact, it is necessary to deal with the random sensor delays where the communication transmission is commonly unreliable and the filtering performance would be degraded. Recently, the problem of linear minimum variance estimation has been investigated in [30] by applying the state augmentation approach for systems with bounded random measurement delays and packet dropouts. In [31], a new optimal filtering scheme has been developed for networked control system subject to random delay and packet dropouts. By applying the quasi Markov-chain approach, the optimal Kalman filtering problem has been studied in [32] for networked control systems with random measurement delays, packet dropouts, and missing measurement. Recently, the recursive filters have been designed in $[33,34]$ for discrete-time systems with different delay rates. However, to the best of authors' knowledge, the problem of the linear optimal filtering has not been thoroughly investigated for discrete state delay systems with randomly multiple sensors delay which constitutes our research motivation.

Motivated by the above discussions, in this paper, we aim to investigate the linear optimal filtering problem for a class of discrete state delay systems measured by multiple sensors with different delay rates. The time delay exists in the system state and the measurement output may experience the random one-step sensor delay probably due to the unreliable communication transmissions. The considered phenomena of multiple measurement delays are characterized by a set of Bernoulli distributed random variables with known conditional probabilities. Based on the MMSE estimation principle, the linear optimal filter is designed which can deal with the effects from the state delay and randomly multiple sensor delays in a unified framework. The main contribution of this paper is to make first attempt to design the optimal filter for state delay systems with randomly multiple sensor delays. Accordingly, a new filtering algorithm is developed and filter gain is obtained recursively by the solutions to the matrix equations. Without resorting to the state augmentation approach, the dimension of the developed filter is the same as the system state and then the proposed filtering algorithm can reduce the computational burden. Finally, a simulation example is shown to verify the feasibility and usefulness of the proposed filtering approach.

Notations. The notations used throughout the paper are standard. $\mathbb{R}^{n}$ denotes the $n$-dimensional Euclidean space. For a matrix $A, A^{T}$ represents its transpose. $\mathbb{E}\{x\}$ represents the expectation of a random variable $x$. I and 0 represent the identity matrix and the zero matrix with appropriate dimensions, respectively. $\operatorname{diag}\left\{X_{1}, X_{2}, \ldots, X_{N}\right\}$ stands for a diagonal matrix with elements $X_{1}, X_{2}, \ldots, X_{N}$ in the diagonal. The Hadamard product is defined as $[T \circ S]_{p \times p}=\left[t_{i j} \times\right.$ $\left.s_{i j}\right]_{p \times p}$ Matrices are assumed to be compatible for algebraic operations if their dimensions are not explicitly stated.

\section{Problem Formulation and Preliminaries}

We consider the following class of linear discrete stochastic systems with state delay:

$$
\begin{gathered}
x_{k+1}=A_{k} x_{k-d}+B_{k} \omega_{k}, \\
z_{k}^{i}=C_{k}^{i} x_{k}+v_{k}^{i}, \\
y_{k}^{i}=\lambda_{k}^{i} z_{k}^{i}+\left(1-\lambda_{k}^{i}\right) z_{k-1}^{i},
\end{gathered}
$$

where $i=1,2, \ldots, N, x_{k} \in \mathbb{R}^{n}$ is the state vector, $d$ is the state delay, $z_{k}^{i} \in \mathbb{R}^{m}$ is the $i$ th actual output, and $y_{k}^{i} \in$ $\mathbb{R}^{m}$ is the measured output of the $i$ th sensor. $\omega_{k} \in \mathbb{R}^{r}$ and $v_{k}^{i} \in \mathbb{R}^{m}$ are uncorrelated zero-mean Gaussian white noises with covariances $Q_{k} \geq 0$ and $R_{k}^{i}>0$, respectively. $A_{k}, B_{k}$, and $C_{k}^{i}$ are known matrices with appropriate dimensions. The random variables $\lambda_{k}^{i}$ obey the Bernoulli distribution and have the following statistical properties:

$$
\begin{gathered}
P\left\{\lambda_{k}^{i}=1\right\}=\mathbb{E}\left\{\lambda_{k}^{i}\right\}=\alpha_{i}, \\
P\left\{\lambda_{k}^{i}=0\right\}=1-\mathbb{E}\left\{\lambda_{k}^{i}\right\}=1-\alpha_{i},
\end{gathered}
$$

where $i=1,2, \ldots, N, \alpha_{i} \in[0,1]$ are known positive scalars, and $\lambda_{k}^{i}$ are uncorrelated with other noise signals.

Remark 1. In model (3), if $\lambda_{k}^{i}=1, y_{k}^{i}=z_{k}^{i}$, it represents that the $i$ th sensor receives successfully the data at time instant $k$. If $\lambda_{k}^{i}=0, y_{k}^{i}=z_{k-1}^{i}$, it stands for the fact that there exists one-step delay.

Setting

$$
\begin{array}{cc}
z_{k}=\left[\begin{array}{c}
z_{k}^{1} \\
z_{k}^{2} \\
\vdots \\
z_{k}^{N}
\end{array}\right], \quad C_{k}=\left[\begin{array}{c}
C_{k}^{1} \\
C_{k}^{2} \\
\vdots \\
C_{k}^{N}
\end{array}\right], \quad v_{k}=\left[\begin{array}{c}
v_{k}^{1} \\
v_{k}^{2} \\
\vdots \\
v_{k}^{N}
\end{array}\right], \\
y_{k}=\left[\begin{array}{c}
y_{k}^{1} \\
y_{k}^{2} \\
\vdots \\
y_{k}^{N}
\end{array}\right], \quad \Lambda_{k}=\left[\begin{array}{llll}
\lambda_{k}^{1} & & \\
& \lambda_{k}^{2} & & \\
& & \ddots & \\
& & & \lambda_{k}^{N}
\end{array}\right],
\end{array}
$$

then, the systems (1)-(3) can be rewritten as follows:

$$
\begin{gathered}
x_{k+1}=A_{k} x_{k-d}+B_{k} \omega_{k}, \\
z_{k}=C_{k} x_{k}+v_{k}, \\
y_{k}=\Lambda_{k} z_{k}+\left(I-\Lambda_{k}\right) z_{k-1} .
\end{gathered}
$$


Substituting (7) into (8), we have

$$
\begin{gathered}
x_{k+1}=A_{k} x_{k-d}+B_{k} \omega_{k} \\
y_{k}=\Lambda_{k} C_{k} x_{k}+\left(I-\Lambda_{k}\right) C_{k-1} x_{k-1}+\Lambda_{k} v_{k}+\left(I-\Lambda_{k}\right) v_{k-1} .
\end{gathered}
$$

It is not difficult to verify that the following statistical properties are true:

$$
\begin{gathered}
\Lambda=\mathbb{E}\left\{\Lambda_{k}\right\}=\operatorname{diag}\left\{\alpha_{1}, \alpha_{2}, \ldots, \alpha_{N}\right\}, \\
R_{k}=\mathbb{E}\left\{v_{k} v_{k}^{T}\right\}=\operatorname{diag}\left\{R_{k}^{1}, R_{k}^{2}, \ldots, R_{k}^{N}\right\}, \\
R_{k-1}=\mathbb{E}\left\{v_{k-1} v_{k-1}^{T}\right\}=\operatorname{diag}\left\{R_{k-1}^{1}, R_{k-1}^{2}, \ldots, R_{k-1}^{N}\right\} .
\end{gathered}
$$

The purpose of this paper is to design the linear optimal filter of state $x_{k+1}$ in the sense of minimum variance for the discrete-time delay stochastic systems (1)-(3) based on the observation sequence $\left\{y_{1}, y_{2}, \ldots, y_{k}\right\}$.

\section{Main Results}

In order to facilitate the subsequent developments, we introduce the following definitions.

Definition 2. Let $\tilde{x}_{i \mid k}=x_{i}-\widehat{x}_{i \mid k}$. Then, define $\Phi_{k(i, j)}=$ $\mathbb{E}\left\{\tilde{x}_{i \mid k} \tilde{x}_{j \mid k}^{T}\right\}$, where $i \neq j$. Particularly, $P_{i \mid k}=\Phi_{k(i, i)}=\mathbb{E}\left\{\tilde{x}_{i \mid k} \tilde{x}_{i \mid k}^{T}\right\}$, when $i=j$. In addition, $\Phi_{k(i, j)}=\Phi_{k(j, i)}^{T}$.

Definition 3. Define $\Theta_{(k, j)}=\mathbb{E}\left\{x_{k} x_{j}^{T}\right\}$, where $k \neq j$. Particularly, $\Theta_{(k, k)}=\mathbb{E}\left\{x_{k} x_{k}^{T}\right\}$, when $k=j$. Also, $\Theta_{(k, j)}=\Theta_{(j, k)}^{T}$. Then, $\Theta_{(k, k)}$ can be calculated as follows:

$$
\Theta_{(k+1, k+1)}=A_{k} \Theta_{(k-d, k-d)} A_{k}^{T}+B_{k} Q_{k} B_{k}^{T} .
$$

Definition 4. Define $\Sigma_{k}^{t}=\Theta_{(k-t, k-d)}$, where $t=0,1, \ldots, d$. Then, $\Sigma_{k}^{t}$ can be calculated as follows:

$$
\Sigma_{k}^{t}=\Theta_{(k-t, k-d)}=A_{k-t-1} \Theta_{(k-t-1-d, k-d)}=A_{k-t-1}\left(\Sigma_{k-t-1}^{d-t-1}\right)^{T},
$$

where $\Sigma_{k}^{d}=\Theta_{(k-d, k-d)}$ can be computed by (12).

Definition 5. Define a series of the matrices as follows:

$$
\begin{gathered}
\Gamma_{k}^{i}=\mathbb{E}\left\{\varepsilon_{k} x_{k-i}^{T}\right\}, \quad Q_{\varepsilon_{k}}=\mathbb{E}\left\{\varepsilon_{k} \varepsilon_{k}^{T}\right\}, \\
K_{k}^{i}=\mathbb{E}\left\{x_{k-i} \varepsilon_{k}^{T}\right\} Q_{\varepsilon_{k}}^{-1}=\left(\Gamma_{k}^{i}\right)^{T} Q_{\varepsilon_{k}}^{-1}, \\
\Psi_{k}^{i}=\Phi_{k(k, k-i+1)}, \quad \Pi_{(k, j)}^{\mu}=\mathbb{E}\left\{x_{k+1-j} x_{k-j-\mu}^{T}\right\}, \\
\Omega_{(k, j)}^{\mu}=\Phi_{k(k+1-j, k-j-\mu)}, \quad \Xi_{k}^{i}=\Phi_{k(k-d, k-i+1)},
\end{gathered}
$$

where $\varepsilon_{k}=y_{k}-\widehat{y}_{k \mid k-1}$ is the innovation sequence.

Next, we are ready to introduce the following lemmas which will be used for the further developments.
Lemma 6. $\Psi_{k}^{i}$ and $\Xi_{k}^{i}$ satisfy the following recursive equation:

$$
\begin{gathered}
\Psi_{k}^{i}=A_{k-1} \Xi_{k-1}^{i-1}-K_{k} \Gamma_{k}^{i-1}, \quad i=2, \ldots, d+1, \\
\Xi_{k}^{i}= \begin{cases}\left(\Psi_{k-d+1}^{d+2-i}\right)^{T}-\sum_{\rho=0}^{i-2} K_{k-\rho}^{d-\rho} \Gamma_{k}^{i-\rho-1}, & i=2, \ldots, d, \\
P_{k-d \mid k}, & i=d+1,\end{cases}
\end{gathered}
$$

where $K_{k}^{0}=K_{k}, \Psi_{k}^{1}=P_{k \mid k}$, and $\Xi_{k}^{1}=\left(\Psi_{k}^{d+1}\right)^{T}$.

Proof. From (10), we can define the innovation sequence by the projection theory as follows:

$$
\begin{aligned}
\varepsilon_{k}= & y_{k}-\Lambda C_{k} \widehat{x}_{k \mid k-1}-(I-\Lambda) C_{k-1} \widehat{x}_{k-1 \mid k-1} \\
= & \left(\Lambda_{k}-\Lambda\right) C_{k} x_{k}+\Lambda C_{k} \tilde{x}_{k \mid k-1} \\
& -\left(\Lambda_{k}-\Lambda\right) C_{k-1} x_{k-1}+(I-\Lambda) C_{k-1} \tilde{x}_{k-1 \mid k-1} \\
& +\Lambda_{k} v_{k}+\left(I-\Lambda_{k}\right) v_{k-1} .
\end{aligned}
$$

By the definitions of $\Psi_{k}^{i}$ and $\Xi_{k}^{i}$, the following equations can be obtained:

$$
\Psi_{k}^{1}=P_{k \mid k}, \quad \Xi_{k}^{1}=\left(\Psi_{k}^{d+1}\right)^{T}, \quad \Xi_{k}^{d+1}=P_{k-d \mid k} .
$$

Subsequently, by employing the projection theory, the recursive equations can be obtained as follows:

$$
\begin{aligned}
\widehat{x}_{k \mid k} & =\widehat{x}_{k \mid k-1}+K_{k} \varepsilon_{k}, \\
K_{k} & =\mathbb{E}\left\{x_{k} \varepsilon_{k}^{T}\right\}\left[\mathbb{E}\left\{\varepsilon_{k} \varepsilon_{k}^{T}\right\}\right]^{-1}, \\
\widehat{x}_{k-i+1 \mid k} & =\widehat{x}_{k-i+1 \mid k-1}+K_{k}^{i-1} \varepsilon_{k}, \\
K_{k}^{i-1} & =\mathbb{E}\left\{x_{k-i+1} \varepsilon_{k}^{T}\right\}\left[\mathbb{E}\left\{\varepsilon_{k} \varepsilon_{k}^{T}\right\}\right]^{-1} .
\end{aligned}
$$

Then, the following equation can be obtained by (19) and (20):

$$
\begin{aligned}
\Phi_{k(k, k-i+1)}= & \Phi_{k-1(k, k-i+1)}-K_{k} \mathbb{E}\left\{\varepsilon_{k} \tilde{x}_{k-i+1 \mid k-1}^{T}\right\} \\
& -\mathbb{E}\left\{\widetilde{x}_{k \mid k-1} \varepsilon_{k}^{T}\right\}\left(K_{k}^{i-1}\right)^{T}+K_{k} \mathbb{E}\left\{\varepsilon_{k} \varepsilon_{k}^{T}\right\}\left(K_{k}^{i-1}\right)^{T} .
\end{aligned}
$$

Noting that the following fact is true.

$$
\begin{gathered}
\mathbb{E}\left\{\Lambda_{k}-\Lambda\right\}=0, \quad \widehat{x}_{k-i+1 \mid k-1} \perp \tilde{x}_{k \mid k-1}, \\
\widehat{x}_{k-i+1 \mid k-1} \perp \widetilde{x}_{k-1 \mid k-1} .
\end{gathered}
$$

We can establish the following equation:

$$
\begin{aligned}
\mathbb{E}\left\{\varepsilon_{k} \tilde{x}_{k-i+1 \mid k-1}^{T}\right\} & =\mathbb{E}\left\{\varepsilon_{k}\left(\widehat{x}_{k-i+1 \mid k-1}+\tilde{x}_{k-i+1 \mid k-1}\right)^{T}\right\} \\
& =\mathbb{E}\left\{\varepsilon_{k} x_{k-i+1}^{T}\right\}=\Gamma_{k}^{i-1} .
\end{aligned}
$$

Substituting $K_{k}$ of (19) into (21) leads to

$$
\Phi_{k(k, k-i+1)}=\Phi_{k-1(k, k-i+1)}-K_{k} \Gamma_{k}^{i-1} .
$$


Similarly, we have

$$
\Phi_{k-\rho(k-d, k-i+1)}=\Phi_{k-\rho-1(k-d, k-i+1)}-K_{k-\rho}^{d-\rho} \Gamma_{k-\rho}^{i-\rho-1} .
$$

From (9), we have

$$
x_{k}=A_{k-1} x_{k-d-1}+B_{k-1} \omega_{k-1} .
$$

Taking projection on both sides of (26), one has

$$
\widehat{x}_{k \mid k-1}=A_{k-1} \widehat{x}_{k-d-1 \mid k-1} .
$$

Subtracting both sides of (27) by (26) yields

$$
\tilde{x}_{k \mid k-1}=A_{k-1} \tilde{x}_{k-d-1 \mid k-1}+B_{k-1} \omega_{k-1} .
$$

Since $\omega_{k-1} \perp \tilde{x}_{k-i+1 \mid k-1}$, the following equation can be derived:

$$
\Phi_{k-1(k, k-i+1)}=A_{k-1} \Phi_{k-1(k-d-1, k-i+1)} .
$$

By the definitions of $\Psi_{k}^{i}$ and $\Xi_{k}^{i}$, substituting (29) into (24) yields

$$
\Psi_{k}^{i}=A_{k-1} \Xi_{k-1}^{i-1}-K_{k} \Gamma_{k}^{i-1} .
$$

According to (25), we can obtain the following equation:

$$
\Xi_{k}^{i}=\left(\Psi_{k-d+1}^{d+2-i}\right)^{T}-\sum_{\rho=0}^{i-2} K_{k-\rho}^{d-\rho} \Gamma_{k}^{i-\rho-1}, \quad i=2, \ldots, d .
$$

Therefore, (15) can be derived by (18) and (30). Moreover, it follows from (18) and (31) that (16) holds. Then, the proof of this lemma is complete.

Lemma 7. For $j=0,1$ and $\mu=0,1, \ldots, d-1, \Pi_{(k, j)}^{\mu}$ and $\Omega_{(k, j)}^{\mu}$ satisfy the following recursive equations:

$$
\begin{gathered}
\Pi_{(k, j)}^{\mu}=A_{k-j}\left(\sum_{k-j}^{\mu}\right)^{T}, \\
\Omega_{(k, j)}^{\mu}= \begin{cases}A_{k} \Xi_{k}^{\mu+1}, & j=0, \\
\Psi_{k}^{\mu+2}, & j=1,\end{cases}
\end{gathered}
$$

where $\Sigma_{k-j}^{\mu}$ is computed by (13) and $\Psi_{k}^{\mu+2}$ and $\Xi_{k}^{\mu+1}$ are calculated by (15) and (16), respectively.

Proof. From (9), we have

$$
x_{k+1-j}=A_{k-j} x_{k-j-d}+B_{k-j} \omega_{k-j} .
$$

By the fact that $x_{k-j-\mu} \perp \omega_{k-j}$ as well as the definitions of $\Pi_{(k, j)}^{\mu}$ and $\Sigma_{k}^{t}$, we have

$$
\begin{gathered}
\mathbb{E}\left\{x_{k+1-j} x_{k-j-\mu}^{T}\right\}=A_{k-j} \mathbb{E}\left\{x_{k-j-d} x_{k-j-\mu}^{T}\right\}, \\
\Pi_{(k, j)}^{\mu}=A_{k-j} \Theta_{(k-j-d, k-j-\mu)}=A_{k-j}\left(\Sigma_{k-j}^{\mu}\right)^{T} .
\end{gathered}
$$

Thus, (32) is true.
At the same time, by the definition of $\Omega_{(k, j)}^{\mu}$, we have

$$
\begin{array}{ll}
\Omega_{(k, 0)}^{\mu}=\Phi_{k(k+1, k-\mu)}, & j=0, \\
\Omega_{(k, 1)}^{\mu}=\Phi_{k(k, k-1-\mu)}, & j=1 .
\end{array}
$$

For (36), by the same idea of (29) and the definition of $\Xi_{k}^{i}$, it can be obtained that

$$
\Omega_{(k, 0)}^{\mu}=\Phi_{k(k+1, k-\mu)}=A_{k} \Phi_{k(k-d, k-\mu)}=A_{k} \Xi_{k}^{\mu+1} .
$$

For (37), by the definition of $\Psi_{k}^{i}$, it can be obtained that

$$
\Omega_{(k, 1)}^{\mu}=\Phi_{k(k, k-1-\mu)}=\Psi_{k}^{\mu+2}
$$

Therefore, it can be shown that (33) holds according to (38) and (39). The proof of this lemma is complete.

Lemma 8. For $j=0,1$, one has

$$
\Phi_{k-1(k-j, k-i)}= \begin{cases}A_{k-1} \Xi_{k-1}^{i}, & j=0 \\ \Psi_{k-1}^{i}, & j=1 .\end{cases}
$$

Proof. When $j=0$, by the same line of (29) and the definition of $\Xi_{k}^{i}$, we obtain

$$
\Phi_{k-1(k, k-i)}=A_{k-1} \Phi_{k-1(k-d-1, k-i)}=A_{k-1} \Xi_{k-1}^{i}
$$

When $j=1$, by the definition of $\Psi_{k}^{i}$, we have

$$
\Phi_{k-1(k-1, k-i)}=\Psi_{k-1}^{i} .
$$

Therefore, it follows from (41) and (42) that (40) is true. The proof of this lemma is complete.

Lemma 9 (see [35]). Let $T=\left[t_{i j}\right]_{p \times p}$ be a real matrix and $S=\operatorname{diag}\left\{s_{1}, s_{2}, \ldots, s_{p}\right\}$ a diagonal random matrix. Then

$$
\mathbb{E}\left\{S T S^{T}\right\}=\left[\begin{array}{cccc}
\mathbb{E}\left\{s_{1}^{2}\right\} & \mathbb{E}\left\{s_{1} s_{2}\right\} & \cdots & \mathbb{E}\left\{s_{1} s_{p}\right\} \\
\mathbb{E}\left\{s_{2} s_{1}\right\} & \mathbb{E}\left\{s_{2}^{2}\right\} & \cdots & \mathbb{E}\left\{s_{2} s_{p}\right\} \\
\vdots & \vdots & \ddots & \vdots \\
\mathbb{E}\left\{s_{p} s_{1}\right\} & \mathbb{E}\left\{s_{p} s_{2}\right\} & \cdots & \mathbb{E}\left\{s_{p}^{2}\right\}
\end{array}\right] \circ T,
$$

where $\circ$ is the Hadamard product.

Now, we are ready to design the linear recursive optimal filter for systems (9)-(10) by employing the observation sequence $\left\{y_{1}, y_{2}, \ldots, y_{k}\right\}$. Based on the above lemmas and motivated by [22], we have the following theorem. 
Theorem 10. The recursive optimal filter for systems (9)-(10) is given as follows:

$$
\begin{aligned}
\widehat{x}_{k+1 \mid k+1}= & \widehat{x}_{k+1 \mid k}+K_{k+1} \varepsilon_{k+1}, \\
\varepsilon_{k+1}= & y_{k+1}-\Lambda C_{k+1} \widehat{x}_{k+1 \mid k}-(I-\Lambda) C_{k} \widehat{x}_{k \mid k} \\
K_{k+1}= & {\left[P_{k+1 \mid k} C_{k+1}^{T} \Lambda+A_{k} \Xi_{k}^{1} C_{k}^{T}(I-\Lambda)\right] Q_{\varepsilon_{k+1}}^{-1} } \\
Q_{\varepsilon_{k+1}}= & \Lambda C_{k+1} P_{k+1 \mid k} C_{k+1}^{T} \Lambda+(I-\Lambda) C_{k} P_{k \mid k} C_{k}^{T}(I-\Lambda) \\
& +\Lambda C_{k+1} \Omega_{(k, 0)}^{0} C_{k}^{T}(I-\Lambda) \\
& +\left(\Lambda C_{k+1} \Omega_{(k, 0)}^{0} C_{k}^{T}(I-\Lambda)\right)^{T} \\
& +\Lambda R_{k+1} \Lambda+(I-\Lambda) R_{k}(I-\Lambda) \\
& +\mathscr{H}_{1}+\mathscr{H}_{2}-\mathscr{H}_{3}-\mathscr{H}_{3}^{T}, \\
P_{k+1 \mid k+1}= & P_{k+1 \mid k} \\
& -K_{k+1}\left[\Lambda C_{k+1} P_{k+1 \mid k}+(I-\Lambda) C_{k}\left(A_{k} \Xi_{k}^{1}\right)^{T}\right] \\
\widehat{x}_{k+1 \mid k}= & A_{k} \widehat{x}_{k-d \mid k}, \\
P_{k+1 \mid k}= & A_{k} P_{k-d \mid k} A_{k}^{T}+B_{k} Q_{k} B_{k}^{T}, \\
\widehat{x}_{k-d \mid k}= & \widehat{x}_{k-d \mid k-d}+\sum_{\rho=0}^{d-1} K_{k-\rho}^{d-\rho} \varepsilon_{k-\rho}, \\
P_{k-d \mid k}= & P_{k-d \mid k-d}-\sum_{\rho=0}^{d-1} K_{k-\rho}^{d-\rho} \Gamma_{k-\rho}^{d-\rho},
\end{aligned}
$$

where

$$
\Gamma_{k}^{i}=\left\{\begin{array}{c}
\Lambda C_{k} \Omega_{(k-1,0)}^{0}+(I-\Lambda) C_{k-1} P_{k-1 \mid k-1}, \quad i=1, \\
\Lambda C_{k} \Omega_{(k-1,0)}^{i-1}+(I-\Lambda) C_{k-1} \Omega_{(k-1,1)}^{i-2}, \quad i=2, \ldots, d, \\
\mathscr{H}_{1}=\Lambda(I-\Lambda) \circ C_{k+1} \Theta_{(k+1, k+1)} C_{k+1}^{T}, \\
\mathscr{H}_{2}=\Lambda(I-\Lambda) \circ C_{k} \Theta_{(k, k)} C_{k}^{T}, \\
\mathscr{H}_{3}=\Lambda(I-\Lambda) \circ C_{k+1} \Pi_{(k, 0)}^{0} C_{k}^{T}
\end{array}\right.
$$

and $K_{k-\rho}^{d-\rho}$ is computed by Definition $5, \Gamma_{k-\rho}^{d-\rho}$ is computed by (53), $\Theta_{(k, k)}$ and $\Xi_{k}^{1}$ are calculated by (12) and (16), respectively, $\Pi_{(k, 0)}^{0}$ and $\Omega_{(k, 0)}^{0}$ are computed by Lemma 7 , and $\mathscr{H}_{1}, \mathscr{H}_{2}$, and $\mathscr{H}_{3}$ are calculated by Lemma 9 .

Proof. By the projection formula, we have

$$
\begin{gathered}
\widehat{x}_{k+1 \mid k+1}=\widehat{x}_{k+1 \mid k}+K_{k+1} \varepsilon_{k+1}, \\
K_{k+1}=\mathbb{E}\left\{x_{k+1} \varepsilon_{k+1}^{T}\right\} Q_{\varepsilon_{k+1}}^{-1} .
\end{gathered}
$$

According to (10), we have the innovation equation as follows:

$$
\begin{aligned}
\varepsilon_{k+1}= & y_{k+1}-\Lambda C_{k+1} \widehat{x}_{k+1 \mid k}-(I-\Lambda) C_{k} \widehat{x}_{k \mid k} \\
= & \left(\Lambda_{k+1}-\Lambda\right) C_{k+1} x_{k+1}+\Lambda C_{k+1} \widetilde{x}_{k+1 \mid k} \\
& -\left(\Lambda_{k+1}-\Lambda\right) C_{k} x_{k}+(I-\Lambda) C_{k} \widetilde{x}_{k \mid k} \\
& +\Lambda_{k+1} v_{k+1}+\left(I-\Lambda_{k+1}\right) v_{k} .
\end{aligned}
$$

Note that $\mathbb{E}\left\{\Lambda_{k+1}-\Lambda\right\}=0, v_{k+1}$, and $v_{k}$ are uncorrelated with other terms. Then, we have

$$
\begin{aligned}
Q_{\varepsilon_{k+1}}= & \mathbb{E}\left\{\varepsilon_{k+1} \cdot \varepsilon_{k+1}^{T}\right\} \\
= & \Lambda C_{k+1} P_{k+1 \mid k}\left(\Lambda C_{k+1}\right)^{T} \\
& +(I-\Lambda) C_{k} P_{k \mid k}\left[(I-\Lambda) C_{k}\right]^{T} \\
& +\Lambda C_{k+1} \Phi_{k(k+1, k)}\left[(I-\Lambda) C_{k}\right]^{T} \\
& +\left(\Lambda C_{k+1} \Phi_{k(k+1, k)}\left[(I-\Lambda) C_{k}\right]^{T}\right)^{T} \\
& +\Lambda R_{k+1} \Lambda^{T}+(I-\Lambda) R_{k}(I-\Lambda)^{T} \\
& +\mathscr{H}_{1}+\mathscr{H}_{2}-\mathscr{H}_{3}-\mathscr{H}_{3}^{T},
\end{aligned}
$$

where

$$
\begin{gathered}
\mathscr{H}_{1}=\mathbb{E}\left\{\left(\Lambda_{k+1}-\Lambda\right) C_{k+1} x_{k+1} x_{k+1}^{T} C_{k+1}^{T}\left(\Lambda_{k+1}-\Lambda\right)^{T}\right\}, \\
\mathscr{H}_{2}=\mathbb{E}\left\{\left(\Lambda_{k+1}-\Lambda\right) C_{k} x_{k} x_{k}^{T} C_{k}^{T}\left(\Lambda_{k+1}-\Lambda\right)^{T}\right\}, \\
\mathscr{H}_{3}=\mathbb{E}\left\{\left(\Lambda_{k+1}-\Lambda\right) C_{k+1} x_{k+1} x_{k}^{T} C_{k}^{T}\left(\Lambda_{k+1}-\Lambda\right)^{T}\right\} .
\end{gathered}
$$

By applying Lemma 9 , the following $\mathscr{H}_{1}$ can be obtained:

$$
\begin{aligned}
\mathscr{H}_{1} & =\mathbb{E}\left\{\left(\Lambda_{k+1}-\Lambda\right) C_{k+1} x_{k+1} x_{k+1}^{T} C_{k+1}^{T}\left(\Lambda_{k+1}-\Lambda\right)^{T}\right\} \\
& =\left[\begin{array}{ccc}
\mathbb{E}\left\{\left(\lambda_{k+1}^{1}-\alpha_{1}\right)^{2}\right\} & \cdots & \mathbb{E}\left\{\left(\lambda_{k+1}^{1}-\alpha_{1}\right)\left(\lambda_{k+1}^{N}-\alpha_{N}\right)\right\} \\
\vdots & \ddots & \vdots \\
\mathbb{E}\left\{\left(\lambda_{k+1}^{N}-\alpha_{N}\right)\left(\lambda_{k+1}^{1}-\alpha_{1}\right)\right\} & \cdots & \mathbb{E}\left\{\left(\lambda_{k+1}^{N}-\alpha_{N}\right)^{2}\right\}
\end{array}\right] \circ C_{k+1} \Theta_{(k+1, k+1} C_{k+1}^{T} \\
& =\operatorname{diag}\left\{\alpha_{1}\left(1-\alpha_{1}\right), \alpha_{2}\left(1-\alpha_{2}\right), \ldots, \alpha_{N}\left(1-\alpha_{N}\right)\right\} \circ C_{k+1} \Theta_{(k+1, k+1)} C_{k+1}^{T} \\
& =\left(\operatorname{diag}\left\{\alpha_{1}, \alpha_{2}, \ldots, \alpha_{N}\right\} \operatorname{diag}\left\{1-\alpha_{1}, 1-\alpha_{2}, \ldots, 1-\alpha_{N}\right\}\right) \circ C_{k+1} \Theta_{(k+1, k+1)} C_{k+1}^{T} \\
& =\Lambda(I-\Lambda) \circ C_{k+1} \Theta_{(k+1, k+1)} C_{k+1}^{T} .
\end{aligned}
$$


By the same derivation of $\mathscr{H}_{1}$, we have

$$
\begin{gathered}
\mathscr{H}_{2}=\Lambda(I-\Lambda) \circ C_{k} \Theta_{(k, k)} C_{k}^{T}, \\
\mathscr{H}_{3}=\Lambda(I-\Lambda) \circ C_{k+1} \Pi_{(k, 0)}^{0} C_{k}^{T} .
\end{gathered}
$$

Then, by the definitions of $\Pi_{(k, j)}^{\mu}$ and $\Omega_{(k, j)}^{\mu}, \mathscr{H}_{1}, \mathscr{H}_{2}, \mathscr{H}_{3}$, and (47) can be obtained.

By the orthogonality of the projection and $\mathbb{E}\left\{\Lambda_{k+1}-\Lambda\right\}=$ $0, \widehat{x}_{k+1 \mid k} \perp \tilde{x}_{k+1 \mid k}, \widehat{x}_{k+1 \mid k} \perp \widetilde{x}_{k \mid k}$, we have

$$
\begin{aligned}
\mathbb{E}\left\{x_{k+1} \varepsilon_{k+1}^{T}\right\} & =\mathbb{E}\left\{x_{k+1}\left(\tilde{x}_{k+1 \mid k} C_{k+1}^{T} \Lambda+\tilde{x}_{k \mid k} C_{k}^{T}(I-\Lambda)\right)\right\} \\
& =\mathbb{E}\left\{\tilde{x}_{k+1 \mid k}\left(\tilde{x}_{k+1 \mid k} C_{k+1}^{T} \Lambda+\tilde{x}_{k \mid k} C_{k}^{T}(I-\Lambda)\right)\right\} \\
& =\Phi_{k(k+1, k+1)} C_{k+1}^{T} \Lambda+\Phi_{k(k+1, k)} C_{k}^{T}(I-\Lambda) .
\end{aligned}
$$

Along the same method of the derivation of (29) as well as the definition of $\Xi_{k}^{i}$, we obtain

$$
\Phi_{k(k+1, k)}=A_{k} \Phi_{k(k-d, k)}=A_{k} \Xi_{k}^{1}
$$

Substituting (57), (61), and (62) into (55) yields (46).

On the other hand, according to (54), we have

$$
\tilde{x}_{k+1 \mid k+1}=\tilde{x}_{k+1 \mid k}-K_{k+1} \varepsilon_{k+1} .
$$

And then

$$
\begin{aligned}
P_{k+1 \mid k+1}= & P_{k+1 \mid k}+K_{k+1} Q_{\varepsilon_{k+1}} K_{k+1}^{T}-\mathbb{E}\left\{\tilde{x}_{k+1 \mid k} \varepsilon_{k+1}^{T}\right\} K_{k+1}^{T} \\
& -K_{k+1} \mathbb{E}\left\{\varepsilon_{k+1} \widetilde{x}_{k+1 \mid k}^{T}\right\} .
\end{aligned}
$$

By the same line of (21), (48) can be obtained.

Thirdly, the following equations can be obtained by the same line of the derivation of (27) and (28):

$$
\begin{gathered}
\widehat{x}_{k+1 \mid k}=A_{k} \widehat{x}_{k-d \mid k}, \\
\tilde{x}_{k+1 \mid k}=A_{k} \tilde{x}_{k-d \mid k}+B_{k} \omega_{k} .
\end{gathered}
$$

By the fact that $\omega_{k} \perp \tilde{x}_{k-d \mid k}$, it can be deduced that

$$
P_{k+1 \mid k}=A_{k} P_{k-d \mid k} A_{k}^{T}+B_{k} Q_{k} B_{k}^{T}
$$

Therefore, (49) and (50) can be obtained by (65) and (67).

Subsequently, the following derivations are given to obtain $\widehat{x}_{k-d \mid k}$ and $P_{k-d \mid k}$. By using the projection theory, we have

$$
\widehat{x}_{k-d \mid k}=\widehat{x}_{k-d \mid k-1}+K_{k}^{d} \varepsilon_{k}
$$

Then

$$
P_{k-d \mid k}=P_{k-d \mid k-1}-K_{k}^{d} \Gamma_{k}^{d}
$$

Hence, it follows from (68) and (69) that we have (51) and (52).

$$
\text { Finally, note that }
$$

$$
\Gamma_{k}^{i}=\mathbb{E}\left\{\varepsilon_{k} x_{k-i}^{T}\right\}=\Lambda C_{k} \Phi_{k-1(k, k-i)}+(I-\Lambda) C_{k-1} \Phi_{k-1(k-1, k-i)} .
$$

When $i=1$, we have

$$
\Gamma_{k}^{1}=\Lambda C_{k} \Phi_{k-1(k, k-1)}+(I-\Lambda) C_{k-1} P_{k-1 \mid k-1} .
$$

When $i \neq 1$, by (40) and (33), the following recursive equations can be obtained:

$$
\Phi_{k-1(k, k-i)}=\Omega_{(k-1,0)}^{i-1}, \quad \Phi_{k-1(k-1, k-i)}=\Omega_{(k-1,1)}^{i-2} .
$$

From (70), (71), and (72), we have (53). The proof of this theorem is now complete.

Remark 11. In Theorem 10, the linear recursive optimal filter is designed for the addressed discrete state delay stochastic systems with random multiple sensor delays. A unified framework is established to address complexities from the state delay and the random multiple sensor delays. The proposed filtering algorithm is of a recursive form suitable for online applications. On the other hand, it is worth mentioning that the proposed linear optimal filter can be reduced to the traditional Kalman filter when $\Lambda=I$.

Remark 12. Note that there has not been much work concerning the design of the optimal filter for systems with state delay and randomly multiple sensor delays. It is well known that, due to the uncertain influence of the practical environment, it is the case and more reasonable to deal with the problem of sensor measurements with different delay rates. Hence, we have made the first attempt to tackle the optimal filtering problem for discrete stochastic systems subject to the state time delay and randomly multiple sensor delays with different delay rates. Compared with the existing results, the developed filtering algorithm can better deal with the engineering practice in a more effective way especially for the case of the different delay rates.

According to Theorem 10, a new recursive algorithm can be established to obtain the linear optimal filter for the addressed discrete state time delay stochastic systems. The following algorithm shows how to design the linear optimal recursive filter in Theorem 10. 
Algorithm 13. The steps of the design of the linear optimal recursive filter are shown as follows:

Step 1. Give the following initial values $\Theta_{(-d,-d)}, \ldots, \Theta_{(0,0)}$; $x_{-d}, \ldots, x_{0} ; \quad \widehat{x}_{-d \mid-d}, \ldots, \widehat{x}_{0 \mid 0} ; \quad P_{-d \mid-d}, \ldots, P_{0 \mid 0} ; \quad \Gamma_{0}^{1}, \ldots, \Gamma_{0}^{d} ;$ $\Psi_{0}^{1}, \ldots, \Psi_{0}^{d}$ and $\Xi_{0}^{1}, \ldots, \Xi_{0}^{d}$.

Step 2. In the time period of $[k-d, d]$, by the value of the previous time, $\Phi_{k-1(k-j, k-d)}$ can be computed.

Step 3. Substituting (40) into (53), we can obtain $\Gamma_{k}^{i}$. Also, $K_{k}^{i}$ can be computed by (53).

Step 4. Calculate $\Psi_{k}^{i}$ by substituting (53) into (15). Calculate $\widehat{x}_{k-d \mid k}$ and $P_{k-d \mid k}$ by (51) and (52). Calculate $\widehat{x}_{k+1 \mid k}$ and $P_{k+1 \mid k}$ by substituting (51) and (52) into (49) and (50).

Step 5. Compute $\Theta_{(k, k)}$ by (12). $\Sigma_{k}^{t}$ is calculated by substituting (12) into (13). Then, (32) is computed by (12) and (13).

Step 6. Substituting (15) and (53) into (16), we have $\Xi_{k}^{i}$. Then, (33) is computed by (15) and (16).

Step 7. Calculate $\mathscr{H}_{1}, \mathscr{H}_{2}$ and $\mathscr{H}_{3}$. Substituting $\mathscr{H}_{1}, \mathscr{H}_{2}, \mathscr{H}_{3}$, (50), and (33) into (47), we obtain $Q_{\varepsilon_{k+1}}$.

Step 8. Substituting (16) and (47) into (46) and (48), we have $K_{k+1}$ and $P_{k+1 \mid k+1}$, respectively.

Step 9. Substituting (51), (49), (46) into (45) and (44), we obtain the optimal estimation $\widehat{x}_{k+1 \mid k+1}$.

Remark 14. According to the above algorithm, the filter gain $K_{k+1}$ can be computed recursively. It is worth pointing out that, when deriving the filter gain, additional efforts should be made to derive the terms $P_{k+1 \mid k+1}$ and $Q_{\varepsilon_{k+1}}$ due to the simultaneous consideration of the randomly multiple sensor delays and the state delay. After having obtained these terms, the filter gain $K_{k+1}$ can be constructed and then the estimation $\widehat{x}_{k+1 \mid k+1}$ can be computed. In the following, an illustrative example will be provided to show the feasibility of the proposed filtering scheme.

\section{An Illustrative Example}

In this section, a numerical example is proposed to show the feasibility and effectiveness of the developed main results.

Consider the following linear discrete-time delay stochastic systems:

$$
\begin{gathered}
x_{k+1}=A_{k} x_{k-2}+B_{k} \omega_{k}, \\
z_{k}^{i}=C_{k}^{i} x_{k}+v_{k}^{i}, \\
y_{k}^{i}=\lambda_{k}^{i} z_{k}^{i}+\left(1-\lambda_{k}^{i}\right) z_{k-1}^{i},
\end{gathered}
$$

where

$$
\begin{gathered}
A_{k}=\left[\begin{array}{ccc}
0.35 & -0.15 & 0.15 \\
0.35 & 0 & 0.1 \\
0 & 0.2 & 0.1
\end{array}\right], \quad B_{k}=\left[\begin{array}{c}
1.5 \\
2 \\
2.5
\end{array}\right], \\
C_{k}^{1}=\left[\begin{array}{c}
0.9 \\
0 \\
0
\end{array}\right]^{T}, \quad C_{k}^{2}=\left[\begin{array}{c}
0 \\
0.85 \\
0
\end{array}\right]^{T}, \quad C_{k}^{3}=\left[\begin{array}{c}
0 \\
0 \\
0.8
\end{array}\right]^{T}
\end{gathered}
$$

and $x_{k}=\left[\begin{array}{lll}x_{k, 1} & x_{k, 2} & x_{k, 3}\end{array}\right]^{T}, \omega_{k}$, and $v_{k}^{i}, i=1,2,3$, are uncorrelated zero-mean Gaussian white noises with covariances 0.2 and 0.1 , respectively.

Let

$$
\begin{aligned}
& \Theta_{(-2,-2)}=\Theta_{(-1,-1)}=\Theta_{(0,0)}=\operatorname{diag}\{1,1,1\} \text {, } \\
& P_{-2 \mid-2}=P_{-1 \mid-1}=P_{0 \mid 0}=\operatorname{diag}\{5,5,5\} \text {, } \\
& x_{-2}=\left[\begin{array}{lll}
-0.3 & -0.1 & 0.1
\end{array}\right]^{T}, \quad x_{-1}=\left[\begin{array}{lll}
-0.2 & 0 & 0.2
\end{array}\right]^{T} \text {, } \\
& x_{0}=\left[\begin{array}{lll}
-0.1 & 0.1 & 0.3
\end{array}\right]^{T}, \quad \widehat{x}_{-2 \mid-2}=\left[\begin{array}{lll}
-0.4 & -0.2 & 0
\end{array}\right]^{T} \text {, } \\
& \widehat{x}_{-1 \mid-1}=\left[\begin{array}{lll}
-0.3 & -0.1 & 0.1
\end{array}\right]^{T} \text {, } \\
& \widehat{x}_{0 \mid 0}=\left[\begin{array}{lll}
-0.2 & 0 & 0.2
\end{array}\right]^{T} \text {, } \\
& \Gamma_{0}^{1}=\Gamma_{0}^{2}=\operatorname{diag}\{1,1,1\}, \quad \Psi_{0}^{1}=\Psi_{0}^{2}=\operatorname{diag}\{1,1,1\}, \\
& \Xi_{0}^{1}=\Xi_{0}^{2}=\operatorname{diag}\{1,1,1\}, \quad \mathbb{E}\left\{\lambda_{k}^{1}\right\}=\alpha_{1}=0.95, \\
& \mathbb{E}\left\{\lambda_{k}^{2}\right\}=\alpha_{2}=0.9, \quad \mathbb{E}\left\{\lambda_{k}^{3}\right\}=\alpha_{3}=0.95
\end{aligned}
$$

and let MSE $i$ denote the mean-square error for the estimation of $x_{k, i}$, that is, $(1 / M) \sum_{j=1}^{M}\left(x_{k, i}^{(j)}-\hat{x}_{k \mid k, i}^{(j)}\right)^{2}$, where $i=1,2,3$ and $M$ is the number of simulation test.

According to Theorem 10, the linear optimal recursive filter can be constructed by applying the innovative analysis approach and MMSE estimation principle. The values of the filter gains are given as in Table 1 . The simulations are shown in Figures 1-6. Among them, Figures 1, 2, and 3 plot the $\log (\mathrm{MSE}-i)(i=1,2,3)$ of the proposed filtering algorithm. The actual system states and the newly designed estimation are plotted in Figures 4, 5, and 6. From the simulations, we can see that the proposed filter can estimate the system state well irrespective of the state delay and the occurrence of the randomly multiple sensor delays with different delay rates. The reason is that, when deriving the recursive optimal filter, we have made additional efforts to compensate the effects from the randomly multiple sensor delays and state delay. 
TABLE 1: Filter gains $K_{k+1}$.

\begin{tabular}{lc}
\hline$k=0$ & {$\left[\begin{array}{lll}3.7383 & 0.3829 & -1.2625 \\
3.3140 & 0.4513 & -0.9463 \\
2.8896 & 0.5198 & -0.6302\end{array}\right]$} \\
$k=1 \quad$ & {$\left[\begin{array}{lll}1.2523 & 0.2830 & -0.1191 \\
0.9912 & 0.4258 & 0.1461 \\
0.7301 & 0.5686 & 0.4114\end{array}\right]$} \\
$k=2 \quad$ & {$\left[\begin{array}{lll}0.7535 & 0.2712 & 0.0502 \\
0.4415 & 0.4235 & 0.3401 \\
0.1296 & 0.5758 & 0.6300\end{array}\right]$} \\
$k=3 \quad\left[\begin{array}{ccc}-0.4412 & 0.4183 & 0.6398 \\
0.6148 & 0.3916 & 0.2305 \\
1.6707 & 0.3649 & -0.1789\end{array}\right]$ \\
$k=4 \quad\left[\begin{array}{lll}0.4101 & 0.3031 & 0.1925 \\
0.4747 & 0.4138 & 0.2985 \\
0.5392 & 0.5245 & 0.4045\end{array}\right]$
\end{tabular}

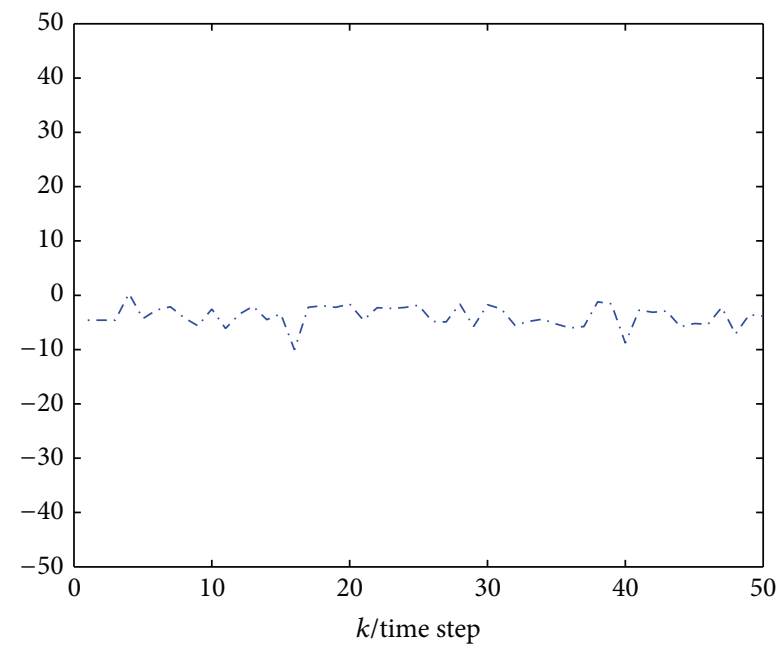

Figure 1: $\log ($ MSE1).

\section{Conclusion}

The problem of linear optimal estimation has been investigated for discrete state delay stochastic systems measured by multiple sensors with different delay rates. Based on the innovative analysis approach and MMSE estimation principle, a new linear optimal filter has been constructed. Compared with the state augmentation approach, the computational burden of the proposed method has been decreased due to the fact that the dimension of the filter is equal to the state vector. Future research topics include the extension of the proposed main results to the filter design for the data-driven systems $[36,37]$ and the networked control systems [3842]. Also, it would be interesting to develop the smoother

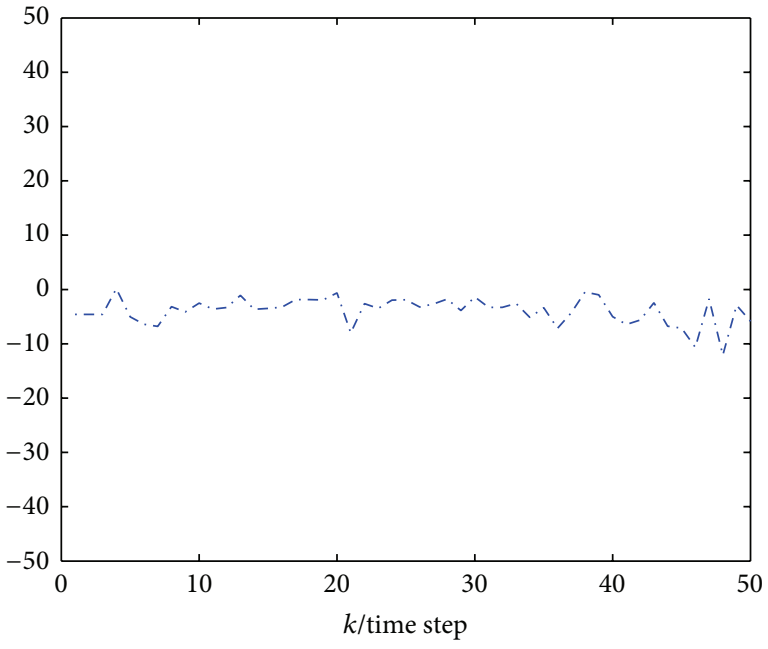

Figure 2: $\log$ (MSE2).

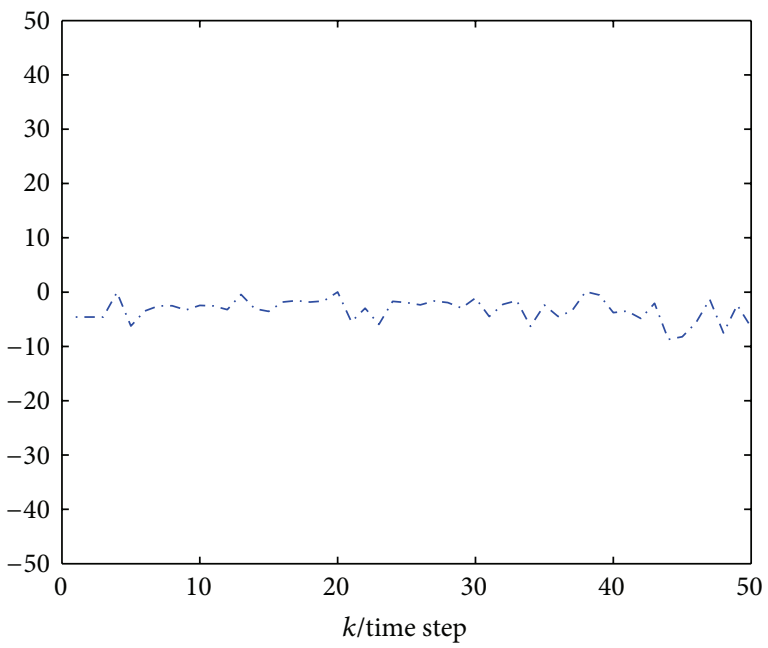

Figure 3: $\log ($ MSE3).

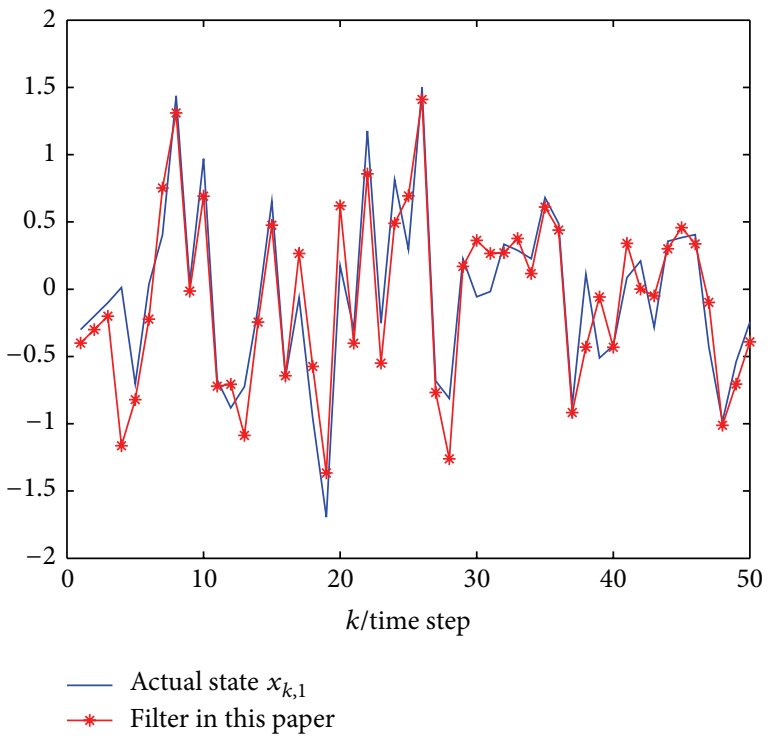

FIGURE 4: The trajectories of $x_{k, 1}$ and $\widehat{x}_{k \mid k, 1}$. 


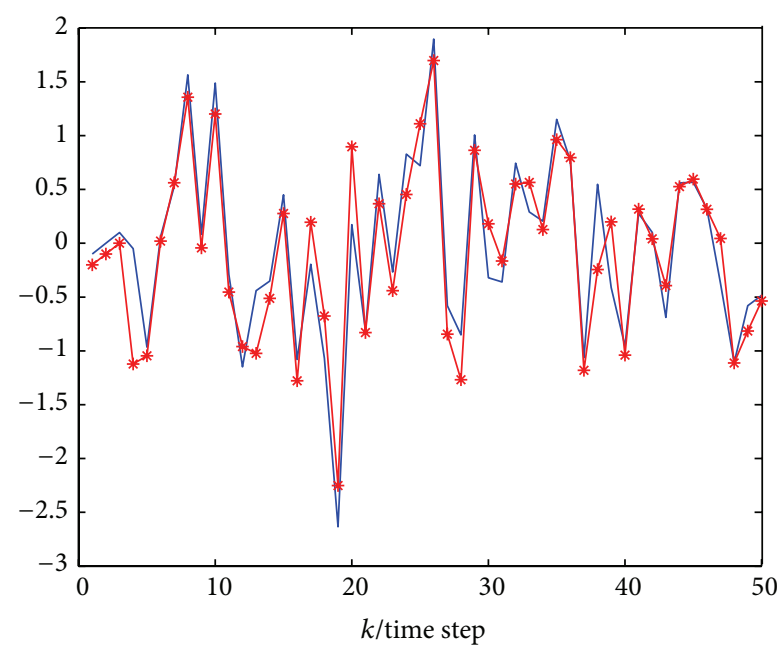

- Actual state $x_{k, 2}$

* Filter in this paper

FIGURE 5: The trajectories of $x_{k, 2}$ and $\widehat{x}_{k \mid k, 2}$.

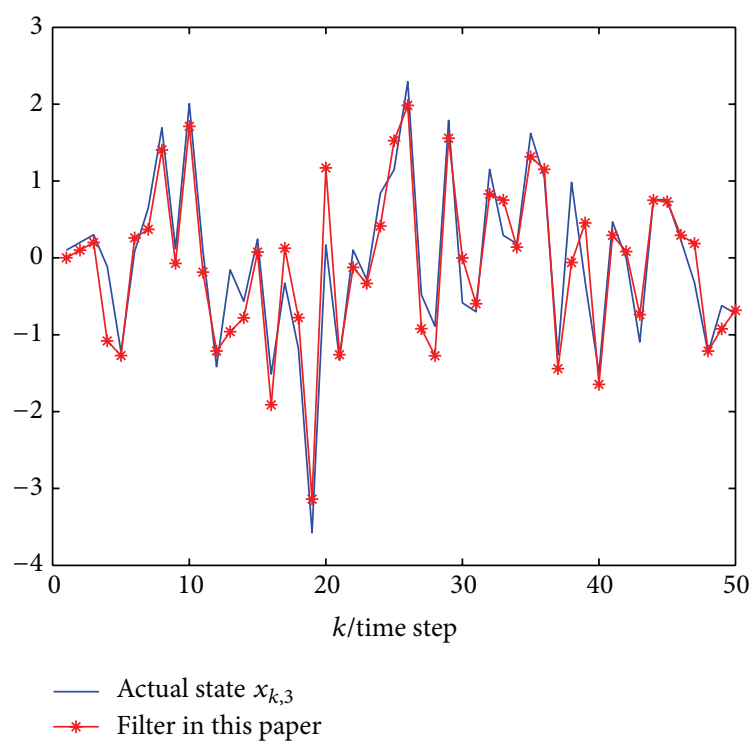

FIGURE 6: The trajectories of $x_{k, 3}$ and $\widehat{x}_{k \mid k, 3}$.

to the addressed networked systems with different delays and discuss the steady-state filter.

\section{Conflict of Interests}

The authors declare that there is no conflict of interests regarding the publication of this paper.

\section{Acknowledgments}

This work was supported in part by the National Natural Science Foundation of China (NSFC) under Grants 11271103 and 11301118 .

\section{References}

[1] H. R. Karimi, M. Zapateiro, and N. Luo, "A linear matrix inequality approach to robust fault detection filter design of linear systems with mixed time-varying delays and nonlinear perturbations," Journal of the Franklin Institute, vol. 347, no. 6, pp. 957-973, 2010.

[2] J. Hu, Z. Wang, H. Gao, and L. K. Stergioulas, "Extended Kalman filtering with stochastic nonlinearities and multiple missing measurements," Automatica, vol. 48, no. 9, pp. 20072015, 2012.

[3] Z. Wang, B. Shen, and X. Liu, " $H_{\infty}$ filtering with randomly occurring sensor saturations and missing measurements," Automatica, vol. 48, no. 3, pp. 556-562, 2012.

[4] Y. Liu, J. Suo, H. R. Karimi, and X. Liu, "A filtering algorithm for maneuvering target tracking based on smoothing spline fitting," Abstract and Applied Analysis, vol. 2014, Article ID 127643, 6 pages, 2014.

[5] Z. Wang, X. Liu, Y. Liu, J. Liang, and V. Vinciotti, "An extended Kalman filtering approach to modeling nonlinear dynamic gene regulatory networks via short gene expression time series," IEEE/ACM Transactions on Computational Biology and Bioinformatics, vol. 6, no. 3, pp. 410-419, 2009.

[6] J. Hu, Z. Wang, H. Dong, and H. Gao, "Recent advances on recursive filtering and sliding mode design for networked nonlinear stochastic systems: a survey," Mathematical Problems in Engineering, vol. 2013, Article ID 646059, 12 pages, 2013.

[7] H. Ahmada and T. Namerikawa, "Extended Kalman filter-based mobile robot localization with intermittent measurements," Systems Science and Control Engineering: An Open Access Journal, vol. 1, no. 1, pp. 113-126, 2013.

[8] X. Kan, H. Shu, and Y. Che, "Asymptotic parameter estimation for a class of linear stochastic systems using Kalman-Bucy filtering," Mathematical Problems in Engineering, vol. 2012, Article ID 342705, 15 pages, 2012.

[9] X. Kan and Z. Wang, "State estimation for discrete-time delayed neural networks with fractional uncertainties and sensor saturations," Neurocomputing, vol. 117, pp. 64-71, 2013.

[10] S. Yin, H. Luo, and S. Ding, "Real-time implementation of faulttolerant control systems with performance optimization," IEEE Transactions on Industrial Electronics, vol. 61, no. 5, pp. 24022411, 2014.

[11] S. Yin, S. Ding, A. Haghani, H. Hao, and P. Zhang, "A comparison study of basic data-driven fault diagnosis and process monitoring methods on the benchmark Tennessee Eastman process," Journal of Process Control, vol. 22, no. 9, pp. 1567-1581, 2012.

[12] P. Shi, Y. Xia, G. P. Liu, and D. Rees, "On designing of slidingmode control for stochastic jump systems," IEEE Transactions on Automatic Control, vol. 51, no. 1, pp. 97-103, 2006.

[13] R. E. Kalman, "A new approach to linear filtering and prediction problems," Transactions of the ASME Journal of the Basic Engineering, vol. 82, pp. 35-45, 1960.

[14] J. Hu, Z. Wang, B. Shen, and H. Gao, "Quantised recursive filtering for a class of nonlinear systems with multiplicative noises and missing measurements," International Journal of Control, vol. 86, no. 4, pp. 650-663, 2013.

[15] J. Hu, Z. Wang, and H. Gao, "Recursive filtering with random parameter matrices, multiple fading measurements and correlated noises," Automatica, vol. 49, no. 11, pp. 3440-3448, 2013. 
[16] C. Yang, Z. Yu, P. Wang, Z. Yu, H. R. Karimi, and Z. Feng, "Robust $l_{2}-l_{\infty}$ filtering for discrete-time delay systems," Mathematical Problems in Engineering, vol. 2013, Article ID 408941, 10 pages, 2013.

[17] X. Lu, H. Zhang, W. Wang, and K. L. Teo, "Kalman filtering for multiple time-delay systems," Automatica, vol. 41, no. 8, pp. 1455-1461, 2005.

[18] J. Hu, Z. Wang, Y. Niu, and L. K. Stergioulas, " $H_{\infty}$ sliding mode observer design for a class of nonlinear discrete time-delay systems: a delay-fractioning approach," International Journal of Robust and Nonlinear Control, vol. 22, no. 16, pp. 1806-1826, 2012.

[19] T. Kaiath and A. H. Sayed, Linear Estimation, Prentice Hall, Upper Saddle River, NJ, USA, 2000.

[20] B. Chen, L. Yu, and W. Zhang, "Optimal filtering for linear discrete state delay systems," in Proceedings of the 29th Chinese Control Conference, pp. 1641-1645, Beijing, China, July 2010.

[21] B. Chen, L. Yu, and W. A. Zhang, "Optimal filtering for linear discrete state delay systems under uncertain observations," Journal of Systems Science and Mathematical Sciences, vol. 30, no. 6, pp. 782-791, 2010.

[22] B. Chen, L. Yu, and W. A. Zhang, "Robust Kalman filtering for uncertain state delay systems with random observation delays and missing measurements," IET Control Theory \& Applications, vol. 5, no. 17, pp. 1945-1954, 2011.

[23] J. Liang, B. Shen, H. Dong, and J. Lam, "Robust distributed state estimation for sensor networks with multiple stochastic communication delays," International Journal of Systems Science, vol. 42, no. 9, pp. 1459-1471, 2011.

[24] J. Hu, D. Chen, and J. Du, "State estimation for a class of discrete nonlinear systems with randomly occurring uncertainties and distributed sensor delays," International Journal of General Systems, vol. 43, no. 3-4, pp. 387-401, 2014.

[25] S. Elmadssia, K. Saadaoui, and M. Benrejeb, "New delaydependent stability conditions for linear systems with delay," Systems Science and Control Engineering: An Open Access Journal, vol. 1, no. 1, pp. 2-11, 2013.

[26] G. Wei, L. Wang, and F. Han, "A gain-scheduled approach to fault-tolerant control for discrete-time stochastic delayed systems with randomly occurring actuator faults," Systems Science and Control Engineering: An Open Access Journal, vol. 1, no. 1, pp. 82-90, 2013.

[27] J. Ma and S. Sun, "Optimal linear estimators for systems with random sensor delays, multiple packet dropouts and uncertain observations," IEEE Transactions on Signal Processing, vol. 59, no. 11, pp. 5181-5192, 2011.

[28] B. Shen, Z. Wang, H. Shu, and G. Wei, " $H_{\infty}$ filtering for nonlinear discrete-time stochastic systems with randomly varying sensor delays," Automatica, vol. 45, no. 4, pp. 1032-1037, 2009.

[29] Z. Wang, H. Dong, B. Shen, and H. Gao, "Finite-horizon $H_{\infty}$ filtering with missing measurements and quantization effects," IEEE Transactions on Automatic Control, vol. 58, no. 7, pp. 17071718, 2013.

[30] S. L. Sun, "Linear minimum variance estimators for systems with bounded random measurement delays and packet dropouts," Signal Processing, vol. 89, no. 7, pp. 1457-1466, 2009.

[31] L. Schenato, "Optimal estimation in networked control systems subject to random delay and packet drop," IEEE Transactions on Automatic Control, vol. 53, no. 5, pp. 1311-1317, 2008.

[32] M. Moayedi, Y. K. Foo, and Y. C. Soh, "Adaptive Kalman filtering in networked systems with random sensor delays, multiple packet dropouts and missing measurements," IEEE Transactions on Signal Processing, vol. 58, no. 3, pp. 1577-1588, 2010.

[33] F. O. Hounkpevi and E. E. Yaz, "Minimum variance generalized state estimators for multiple sensors with different delay rates," Signal Processing, vol. 87, no. 4, pp. 602-613, 2007.

[34] J. Hu, Z. Wang, B. Shen, and H. Gao, "Gain-constrained recursive filtering with stochastic nonlinearities and probabilistic sensor delays," IEEE Transactions on Signal Processing, vol. 61, no. 5, pp. 1230-1238, 2013.

[35] R. A. Horn and C. R. Johnson, Topics in Matrix Analysis, Cambridge University Press, New York, NY, USA, 1991.

[36] S. Yin, G. Wang, and H. R. Karimi, "Data-driven design of robust fault detection system for wind turbines," Mechatronics, 2013.

[37] S. Yin, S. X. Ding, A. H. A. Sari, and H. Hao, "Data-driven monitoring for stochastic systems and its application on batch process," International Journal of Systems Science, vol. 44, no. 7, pp. 1366-1376, 2013.

[38] H. R. Karimi, P. J. Maralani, B. Lohmann, and B. Moshiri, " $H_{\infty}$ control of parameter-dependent state-delayed systems using polynomial parameter-dependent quadratic functions," International Journal of Control, vol. 78, no. 4, pp. 254-263, 2005.

[39] J. Hu, Z. Wang, H. Gao, and L. K. Stergioulas, "Probabilityguaranteed $H_{\infty}$ finite-horizon filtering for a class of nonlinear time-varying systems with sensor saturations," Systems \& Control Letters, vol. 61, no. 4, pp. 477-484, 2012.

[40] H. R. Karimi, "Robust synchronization and fault detection of uncertain master-slave systems with mixed time-varying delays and nonlinear perturbations," International Journal of Control, Automation and Systems, vol. 9, no. 4, pp. 671-680, 2011.

[41] Z. Wang, D. Ding, H. Dong, and H. Shu, " $H_{\infty}$ consensus control for multi-agent systems with missing measurements: the finitehorizon case," Systems \& Control Letters, vol. 62, no. 10, pp. 827836, 2013.

[42] H. R. Karimi, "Robust $H_{\infty}$ filter design for uncertain linear systems over network with network-induced delays and output quantization," Modeling, Identification and Control, vol. 30, no. 1, pp. 27-37, 2009. 


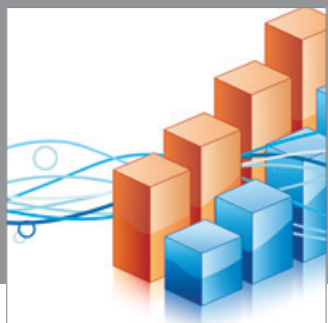

Advances in

Operations Research

mansans

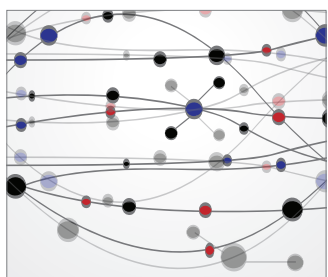

The Scientific World Journal
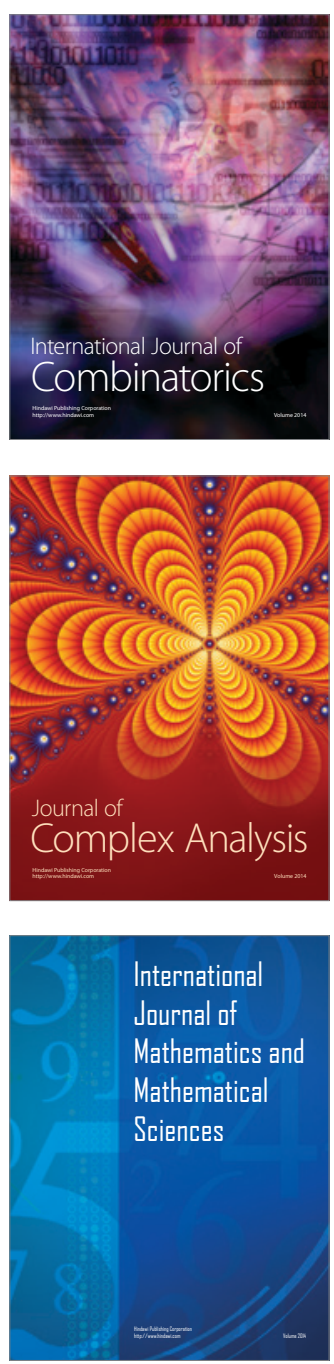
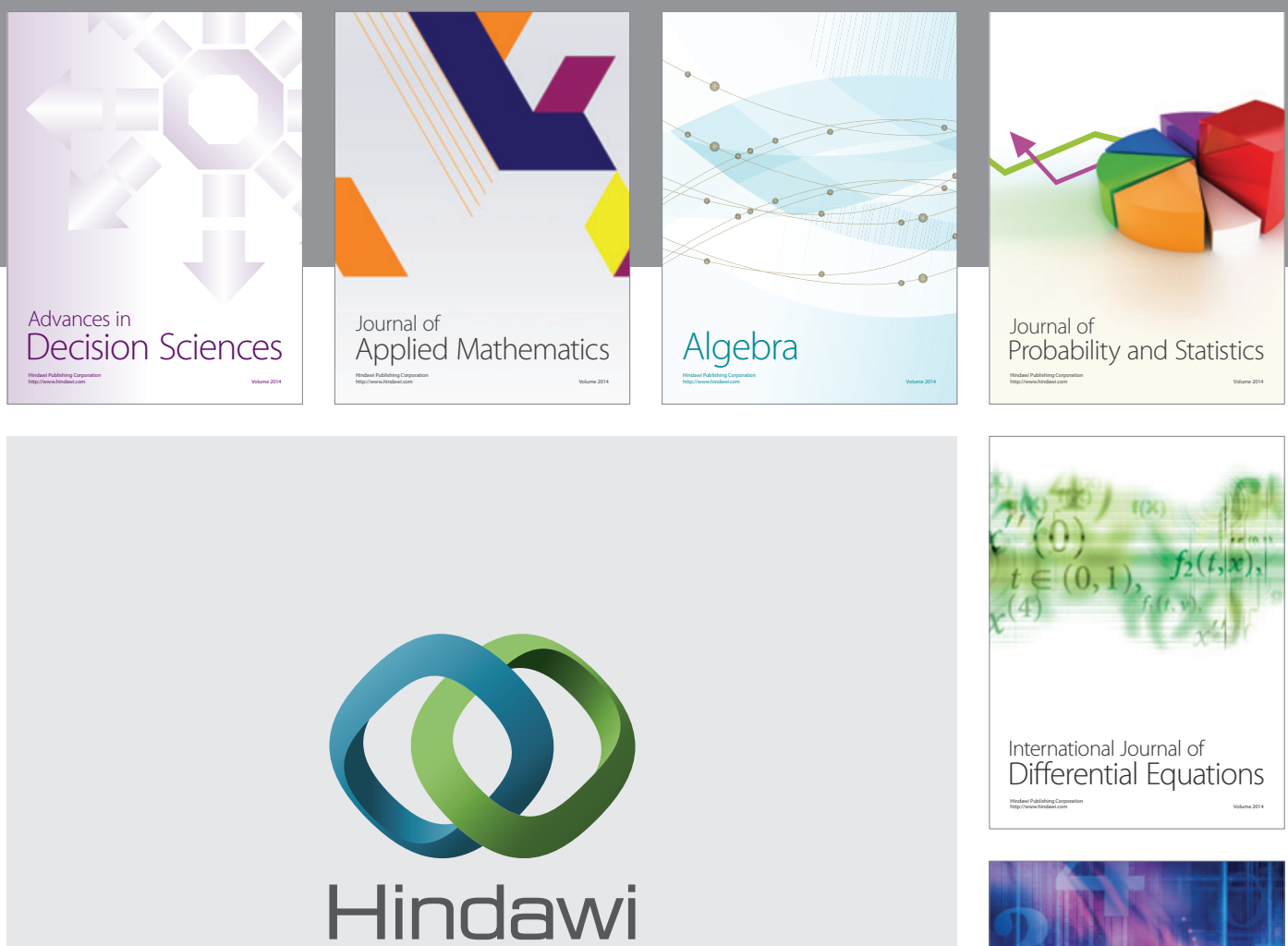

Submit your manuscripts at http://www.hindawi.com
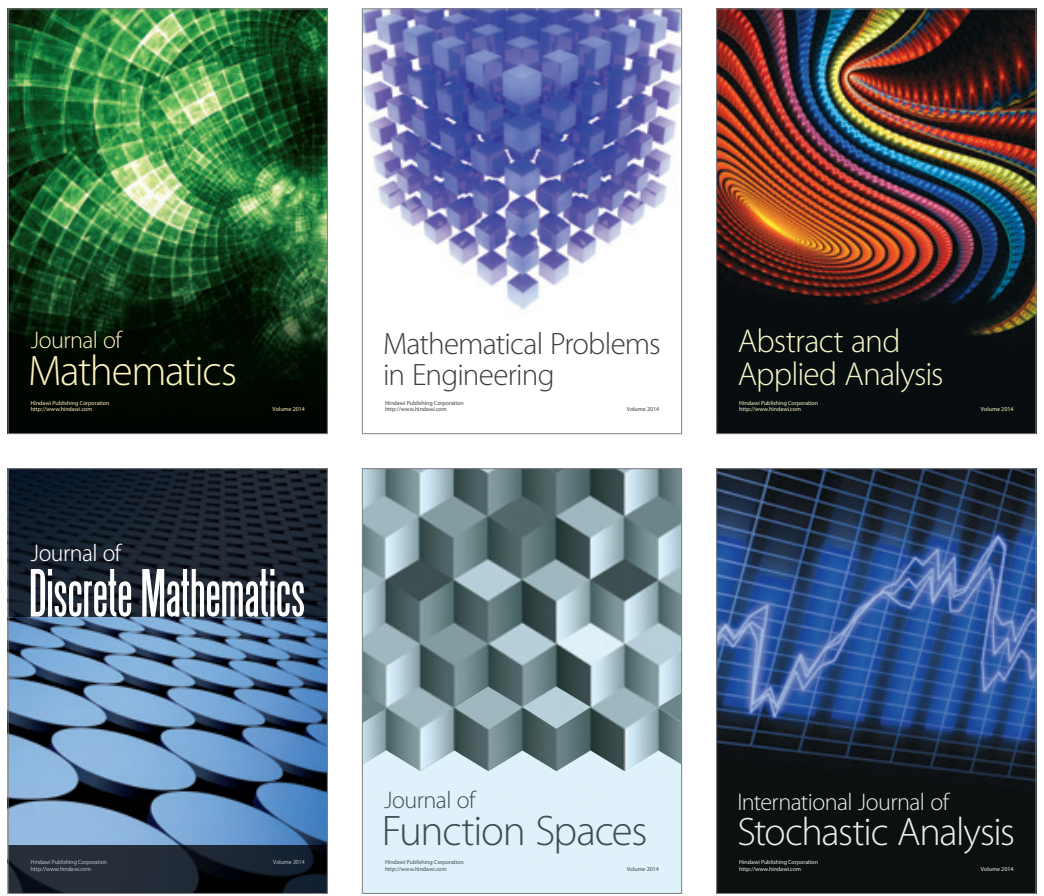

Journal of

Function Spaces

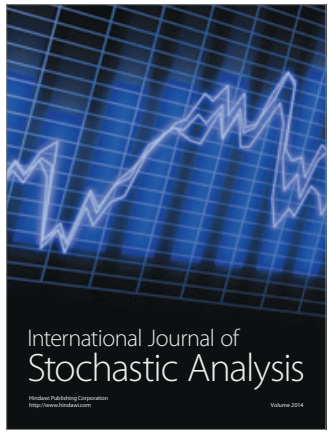

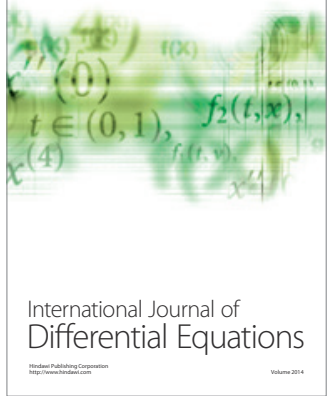
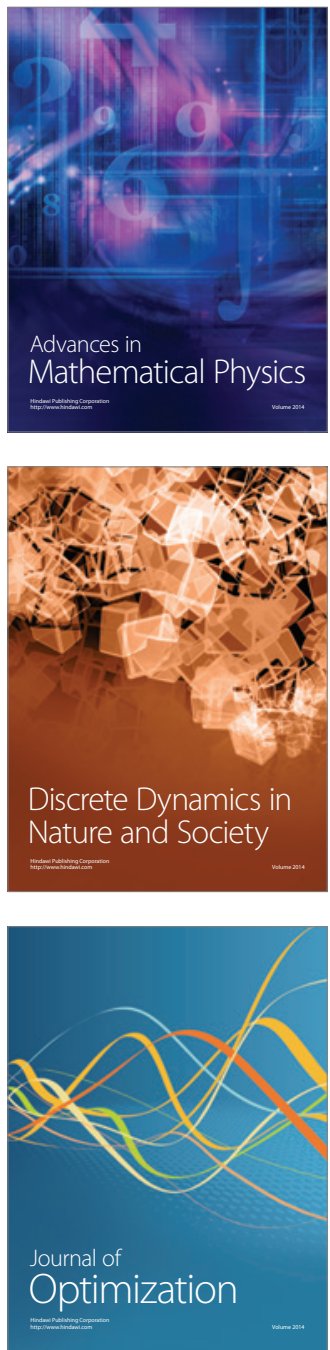